\title{
Les inflammasomes
}

\section{Plates-formes de \\ l'immunité innée}

Yvan Jamilloux ${ }^{1,2,3,4,5}$, Thomas Henry ${ }^{2,3,4,5}$

> L'homme est constamment exposé à des pathogènes et a développé un système immunitaire extrêmement complexe pour s'en défendre. Le système immunitaire inné constitue la première ligne de défense de l'organisme. II a évolué pour reconnaître des motifs microbiens conservés (pathogen-associated molecular pattern, PAMP) grâce un ensemble de récepteurs (pattern recognition receptor, PRR). La reconnaissance de la plupart des PAMP par les PRR entraîne une réponse transcriptionnelle qui se traduit par la néosynthèse de centaines de protéines. L'inflammasome, quant à lui, est un complexe de l'immunité innée qui, en réponse à la détection de motifs microbiens ou de signaux de danger par certains PRR, conduit au contraire à l'activation spécifique d'une caspase inflammatoire, la caspase-1. La caspase-1 activée déclenche la sécrétion de cytokines pro-inflammatoires, telles que l'interleukine (IL)- $1 \beta$ et I'IL-18, ainsi qu'une mort cellulaire programmée appelée pyroptose. Dans cette revue, nous décrivons les récepteurs de l'inflammasome et leurs ligands, l'assemblage et le fonctionnement de ce complexe protéique, ainsi que le rôle de l'inflammasome dans les infections bactériennes et virales. <

L'étude des mécanismes de l'immunité innée a connu un regain d'intérêt depuis la découverte des récepteurs Tolllike (TLR), il y a une vingtaine d'années [49]. Par la suite, la mise en évidence d'autres récepteurs de l'immunité innée (les RIG-l-like receptors ou RLR, les C-type lectin receptors ou CLR, et les nucleotide-binding domain and leucin-rich repeats containing receptors ou NLR) a permis de décrypter les mécanismes de détection des pathogènes via la reconnaissance rapide de motifs microbiens conservés, nommés PAMP. La plupart de ces motifs étant conservés dans les espèces non pathogènes, ils sont aussi

Vignette (Photo @ Inserm - Odile Filhol).

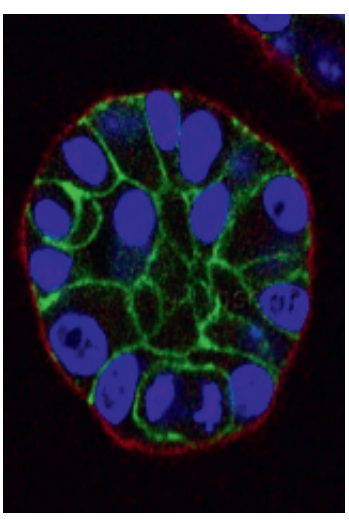

\begin{abstract}
${ }^{1}$ Service de médecine interne, centre hospitalo-universitaire de la Croix-Rousse,

103, Grande rue de la Croix Rousse, 69004 Lyon, France ;

${ }^{2}$ Centre international de recherche en infectiologie (CIRI), université de Lyon, Lyon, France ; ${ }^{3}$ Inserm U1111, 21, avenue Tony Garnier, 69007 Lyon, France; ${ }^{4}$ CNRS, UMR 5308, Lyon, France ; ${ }^{5}$ École normale supérieure, Lyon, France.
\end{abstract}

yvanjamilloux@hotmail.com

nommés MAMP (microbe-associated molecular patterns) [1]. Les récepteurs de ces PAMP ont été nommés PRR (pattern recognition receptors). De manière plus générale, ces PRR peuvent être activés par des signaux de danger, regroupés sous la dénomination de DAMP (damage-associated molecular patterns) [2]. Cette reconnaissance de motif permet à un nombre limité de PRR de reconnaître une grande diversité de pathogènes. Cette large capacité de reconnaissance est augmentée par la diversité des localisations de ces PRR. Ainsi, les TLR et les CLR surveillent le milieu extracellulaire et les compartiments endosomaux, tandis que les RLR et les NLR scrutent la présence de motifs microbiens ou de signaux de danger au sein du cytoplasme cellulaire. En 2002, Jurg Tschopp et al. [3] ont découvert un macrocomplexe moléculaire comportant un PRR d'un type nouveau et l'ont désigné inflammasome. Contrairement aux PRR identifiés précédemment, qui induisent une réponse transcriptionnelle via l'activation de facteurs de transcription tels que NF-KB ou IRF3/7 (IFN regulatory factor $3 / 7$ ) et la synthèse de centaines de protéines, la mise en jeu de l'inflammasome entraîne spécifiquement l'activation d'une caspase inflammatoire, la caspase-1 (Encadré 1). La caspase-1 a un double rôle : le clivage protéolytique de procytokines inflammatoires comme l'IL-1 $\beta$ et I'IL-18, qui permet leur maturation et leur sécrétion, et le déclenchement d'une mort cellulaire hyper-inflammatoire, la pyroptose [4].

Les inflammasomes sont donc définis comme des plates-formes activatrices de la caspase-1. La dénomination de chacun d'entre eux résulte du nom du PRR impliqué. Cette revue décrit les différents inflammasomes identifiés, leurs modes d'activation et leurs implications en pathologie infectieuse.

\section{Description des inflammasomes}

L'inflammasome est un complexe macromoléculaire qui se forme par oligomérisation d'un récepteur, d'un adaptateur et de la caspase-1, 


\section{Caspases inflammatoires et caspases apoptotiques}

Les caspases sont des protéases à cystéine. Elles sont classées en deux groupes : les caspases apoptotiques qui déclenchent l'apoptose (une mort cellulaire largement silencieuse du point de vue immunologique), et les caspases inflammatoires qui déclenchent une mort de type nécrotique souvent accompagnée de la libération de cytokines. Chez l'homme, les caspases inflammatoires sont les caspases-1, -4, -5 et -12. Le rôle de la caspase-1 dans l'inflammation est bien décrit. Le rôle des caspases -4 et -5 est moins bien connu et est extrapolé à partir du rôle de l'homologue murin de ces caspases : la caspase-1l. La caspase-11 peut activer, de manière indirecte, la caspase-1 ou déclencher la pyroptose de manière indépendante de la caspase-1. La caspase-12 est non fonctionnelle chez la majorité des humains. Dans certaines populations d'Afrique sub-saharienne, une forme longue de caspase-12 est synthétisée et semble, paradoxalement, avoir un rôle anti-inflammatoire.

l'effecteur de ce complexe (Figure 1). L'association de ces différents partenaires se fait via l'interaction entre domaines homotypiques.

\section{Les récepteurs}

Trois familles de récepteurs sont actuellement décrites comme principaux déclencheurs de l'activation de l'inflammasome : les NLR, les ALR (AIM2 [absent in melanoma 2]-like receptors) et les RLR. Ces récepteurs et la cascade inflammasome qui en résulte ont principalement été décrits dans les macrophages et les cellules dendritiques, mais ils sont présents aussi dans d'autres cellules, telles que les polynucléaires neutrophiles, les kératinocytes ou les cellules épithéliales intestinales.

\section{Les récepteurs NLR}

Chez l'homme, 22 récepteurs appartenant à la famille des NLR ont été identifiés. Tous possèdent une structure organisée en domaines [5].

À l'instar des TLR, l'extrémité carboxy-terminale comporte un domaine leucine-rich repeats (LRR) composé d'une répétition caractéristique de 20 à 30 acides aminés, riche en leucine. Ce domaine serait le site d'interaction entre le récepteur et son ligand. La partie centrale, commune à tous les NLR, correspond au domaine NBD (nucleotide binding domain). Ce domaine permet l'oligomérisation dépendante de I'ATP des NLR en hexamères ou heptamères, conférant à l'inflammasome sa forme typique de doughnut (Figure 2). Le domaine LRR possède un rôle d'autorégulation bien connu. En effet, son repliement conformationnel, en dehors de toute stimulation, dissimule le domaine d'oligomérisation. L'activation du récepteur entraîne un changement de conformation et expose le domaine NBD, initiant ainsi la cascade de réactions conduisant à la formation de l'inflammasome.

L'extrémité amino-terminale porte le domaine effecteur des NLR, responsable de la transduction du signal. La nouvelle nomenclature des NLR les regroupe en sous-familles en fonction du domaine effecteur [5]. On distingue ainsi les NLRP qui ont un pyrin domain (PyD), les NLRC, auxquels appartiennent NODI (nucleotide-binding oligomerization domain-containing protein 1), NOD2 et NLRC4/IPAF, qui ont un caspase activation and recruitment domain (CARD), ainsi que NLRB/NAIP (NLR family, apoptosis inhibitory protein) qui a un baculoviral inhibitor of apoptosis protein repeat (BIR).

À l'heure actuelle, sept NLR ont été identifiés comme inducteurs d'une activité inflammasome. NLRP3 (ou cryopyrine), le plus largement étudié, est impliqué dans la détection de nombreux signaux de danger, et des anomalies intrinsèques de ce récepteur sont la cause de diverses maladies affectant I'homme [6-8]. NLRPI, NLRP7 et les complexes NLRB/NLRC4 ont un rôle plus spécialisé dans la détection de PAMP. NLRPI chez la souris détecte la toxine de l'anthrax. Chez l'homme, NLRPI détecterait un fragment du peptidoglycane bactérien. NLRP7 détecte un lipopeptide des mycoplasmes. Les complexes NLRB/NLRC4 détectent la flagelline sécrétée dans le cytosol et les systèmes de sécrétion de type III des bactéries pathogènes. NLRP6, NLRP12 et NOD2 (un autre récepteur détectant des fragments de peptidoglycane) peuvent activer l'inflammasome, mais leurs rôles restent moins bien caractérisés.

\section{Les récepteurs ALR}

Chez l'homme, la famille des ALR comporte quatre membres: AIM2, IFI16 (interferon $\gamma$-inducible protein 16), MNDA (myeloid cell nuclear differentiation antigen), et IFIX (interferon inducible protein X) [9]. L'extrémité carboxy-terminale des ALR contient un domaine de liaison à l'ADN double-brin appelé HIN200. L'extrémité amino-terminale contient un domaine PyD qui interagit avec le domaine PyD d'autres protéines (par exemple ASC [apoptosis-associated Speck-like protein]) afin de former des macrocomplexes ayant un rôle dans l'inflammation et la mort cellulaire. Seuls AIM2 et IFII6 ont été décrits comme inducteurs de l'inflammasome suite à la détection d'ADN dans le cytoplasme et dans le noyau, respectivement $[10,11]$.

\section{Les récepteurs $\mathbf{R} \mathbf{R}$}

La reconnaissance du récepteur cytoplasmique RIG-I (retinoic acid inducible gene 1 ) comme récepteur de l'inflammasome est récente et reste sujet de débat. La famille des RLR comporte trois membres: RIG-I, MDA5 (melanoma differentiation-associated protein 5), et LGP2. Les RLR comportent un domaine hélicase central responsable de la liaison aux ARN et un domaine régulateur à l'extrémité carboxy-terminale. RIG-I et MDA5 [50,5l] contiennent également deux domaines CARD en amino-terminal, alors que LGP2 n'a pas de CARD. LGP2 pourrait être, en fait, un régulateur des deux autres RLR [12]. RIG-I et MDA5, malgré leurs structures homologues, ne reconnaissent pas les mêmes 


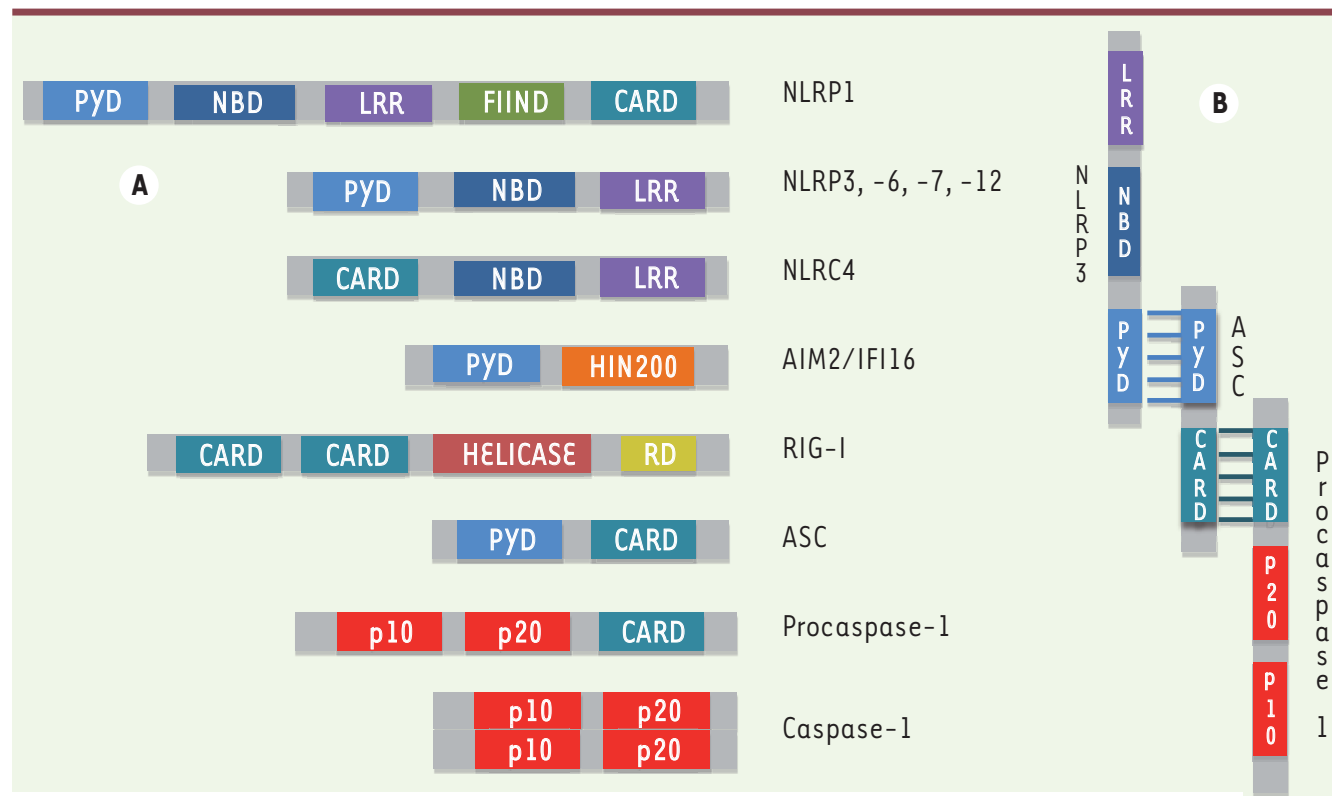

Figure 1. Structure de l'inflammasome. A. Structure des domaines composant l'inflammasome. B. L'inflammasome NLRP3 est composé du récepteur NLRP3, de l'adaptateur ASC et de la procaspase-1. PyD : pyrin domain ; NBD : nucleotide binding domain (permet l'oligomérisation dépendante de l'ATP des NLR) ; LRR : leucine-rich repeats ; FIIND : function to find domain ; CARD : caspase activation and recruitment domain ; RD : repression domain. la transcription du gène codant pour la pro-IL-1 $\beta$. Le second signal est donné par de nombreux activateurs (Tableau I).

L'inflammasome NLRP3 est, par exemple, activé en réponse à de nombreux signaux de danger traduisant des dommages membranaires, et à des modifications de l'homéostasie ionique ou métabolique de la cellule :

- La lyse cellulaire est associée à un relargage de molécules cytosoliques qui peuvent se comporter comme des signaux de

ARN viraux et n'entraînent pas la transduction des mêmes signaux. En effet, si les deux récepteurs sont capables d'activer les facteurs de transcription IRF (interferon regulatory factor) et NFKB, et d'induire secondairement la transcription du gène codant pour l'interféron (IFN) de type 1, seul RIG-I semble capable d'interactions CARD-CARD avec la caspase-1. Cependant, son rôle en tant que récepteur de l'inflammasome nécessite d'être confirmé, notamment son activation indépendamment de NLRP3 [13].

Depuis la découverte pionnière de l'inflammasome NLRP1, le nombre de récepteurs se comportant comme des activateurs de la caspase-1 n'a cessé d'augmenter. En plus de la famille des NLRP, qui contient chez l'homme 14 activateurs potentiels de l'inflammasome, des 4 ALR et des 3 RLR, d'autres récepteurs, tels que la pyrine ou des CLR, pourraient être des activateurs directs de l'inflammasome. Leur pertinence biologique doit cependant être démontrée.

\section{Ligands et activateurs des récepteurs}

La liste des activateurs des inflammasomes s'est considérablement allongée ces dernières années, du fait de l'engouement suscité par les inflammasomes auprès des chercheurs à travers le monde. Les principaux stimulus activateurs des inflammasomes sont détaillés dans le Tableau I.

\section{L'activation de NLRP3}

L'activation de l'inflammasome canonique NLRP3 nécessite la conjonction de deux signaux. Le premier signal, résultant souvent de l'engagement d'un récepteur Toll-like, induit une pré-activation de NLRP3 via une modification post-traductionnelle (déubiquitination) [14], une augmentation de la transcription du gène correspondant, ainsi que danger (DAMP). Ainsi, l'ATP est un messager extracellulaire détecté par le récepteur purinergique $\mathrm{P} 2 \mathrm{X}_{7}$ [52]. L'activation de ce récepteur membranaire entraîne l'ouverture d'un canal protéique (la pannexine) et un efflux d'ions potassium hors de la cellule. La diminution de la concentration intracellulaire en potassium induit un changement de conformation de NLRP3 et l'activation de l'inflammasome NLRP3 [15-17]. De la même manière, des variations de l'homéostasie calcique pourraient participer à la détection des dommages membranaires via l'activation de NLRP3 [18].

- Une autre voie d'activation de NLRP3 a été proposée dans le cas des particules, telles que l'amiante ou la silice $[7,19]$, ou des cristaux d'acide urique [8] (responsables des crises de goutte), de cholestérol [6] ou d'hémozoïne (un cristal dérivé de l'hémoglobine qui se forme lors des infections à Plasmodium falciparum, un agent du paludisme $[54](\rightarrow)$, ou enfin de la protéine $\beta$-amyloïde (maladie d'Alzhei-

$\rightarrow$ Voir la Nouvelle, de K.G. Pellé et al., mer) [20]. Ces cristaux et particules page $\mathbf{9 6 0}$ de ce numéro peuvent entraîner une rupture des lysosomes ou des phagolysosomes associée à un relargage du contenu de ces organites dans le cytosol. En particulier, le relargage de cathepsines (protéases lysosomales) dans le cytosol serait impliqué dans l'activation de NLRP3 [21]. Les bactéries productrices de toxines formant des pores dans la membrane cellulaire, comme par exemple Staphylococcus aureus, activeraient NLRP3 via l'une de ces deux voies de perception des dommages membranaires [22, 23]. 


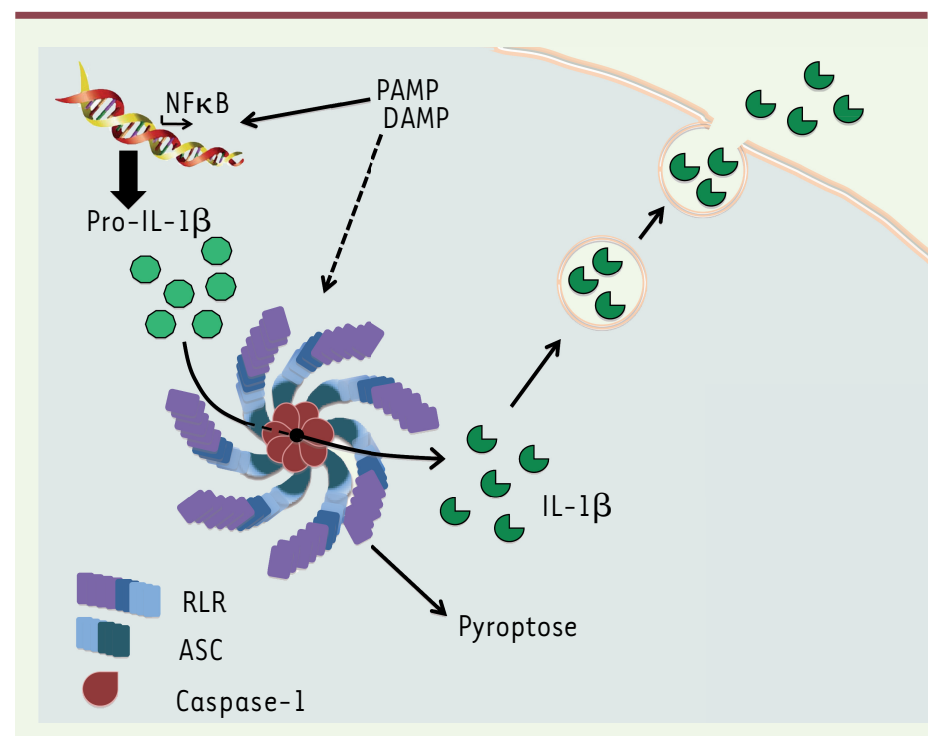

Figure 2. Schéma général du fonctionnement de NLRP3. Après une étape de préactivation, les signaux de danger (DAMP) et/ou les motifs microbiens (PAMP) entraînent la formation de l'inflammasome par interaction entre le récepteur NLRP3, l'adaptateur ASC et l'effecteur, la procaspase-1. L'activation de la caspase-l qui en résulte induit le clivage du prodomaine de la pro-IL-1 $\beta$, la transformant en sa forme bioactive, I'IL-1 $\beta$, qui est ensuite sécrétée dans le milieu extracellulaire.

- Enfin, la mitochondrie semble être au carrefour de nombreux stimulus activant NLRP3. De nombreux stress cellulaires peuvent conduire à la production de dérivés réactifs de l'oxygène (ROS) par la mitochondrie. Ces ROS pourraient constituer le premier signal et rendre compétent NLRP3 [21, 24]. De plus, ils pourraient directement activer NLRP3 en modifiant la thiorédoxine, une protéine sensible aux ROS, en libérant son partenaire d'interaction TXNIP (thioredoxin-interacting protein) qui pourrait alors interagir avec NLRP3 et activer cet inflammasome [25]. De plus, ces ROS, ainsi que d'autres signaux de stress cellulaires, conduisent à la lyse mitochondriale qui entraîne la libération d'ADN mitochondrial oxydé dans le cytosol. Ce dernier serait directement détecté par NLRP3 [26]. Finalement, la mitochondrie intègre les stress métaboliques cellulaires (par exemple la faillite énergétique de la cellule qui active l'inflammasome NLRP3) et peut constituer une plate-forme sur laquelle se formerait l'inflammasome NLRP3.

\section{Transduction du signal et activation de la caspase-1}

L'activation des NLR entraîne leur oligomérisation via le domaine NBD et conduit au regroupement des domaines PYD. La protéine ASC (apoptosis-associated speck-like protein containing a CARD) est ensuite recrutée par interaction PYD-PYD. ASC est une protéine adaptatrice, car elle contient un domaine PYD en amino-terminal (interagissant avec la plupart des récepteurs) et un domaine CARD en carboxy-terminal. Ce dernier est capable d'interagir à son tour avec le CARD de la procaspase-1. La protéine ASC forme également un complexe avec les récepteurs AIM2 et IFI16 lors de leur activation.
En revanche, NLRC4 et RIG-I interagissent directement avec la procaspase-1. Dans le cas de NLRC4 et en fonction du stimulus, l'intensité de l'activation de la caspase-1 peut également nécessiter l'interaction d'ASC ou de NLRCB (ex-NAIP) [27-29].

Le rapprochement conformationnel de deux procaspases-1 dans le complexe ainsi formé entraîne leur clivage autoprotéolytique et leur autoactivation. Sous sa forme active, la caspase-l est un tétramère formé de deux paires de sous-unités, p10 et p20. D'autres caspases pourraient être activées par des complexes inflammasomes non canoniques (Encadré 2). La caspase- 1 est une protéase à cystéine dont les rôles principaux sont la conversion de la pro-IL-1 $\beta$ et de la pro-IL-18 en leurs formes actives par clivage des prodomaines carboxy-terminaux et le déclenchement de la pyroptose.

\section{Les interleukines pro-inflammatoires}

\section{L'interleukine-1 $\beta$}

L'IL-1 $\beta$ est une cytokine majeure de la réponse inflammatoire, connue auparavant sous la dénomination de pyrogène endogène $[30,31]$. Elle exerce de nombreuses fonctions comme l'induction de la fièvre au niveau cérébral ou la stimulation de la moelle osseuse à l'origine d'hyperleucocytose et de thrombocytose. Par ailleurs, elle peut activer directement les lymphocytes, les cellules épithéliales et les cellules endothéliales, ce qui entraîne le recrutement de polynucléaires neutrophiles et de cellules inflammatoires au site de l'infection. L'IL- $1 \beta$ peut aussi induire sa propre expression et l'expression de gènes codant pour d'autres cytokines (tumor necrosis factor- $\alpha$ [TNF- $\alpha]$ ou IL-6), créant ainsi une véritable cascade inflammatoire.

Du fait de sa puissance pro-inflammatoire et de ses effets délétères potentiels, la synthèse, la sécrétion et l'activité de l'IL- $1 \beta$ sont très finement régulées par différents processus : nécessité d'un signal activateur (priming) pour déclencher la transcription dépendante de NFKB de la pro-IL-1 $\beta$ sous forme inactive; clivage régulé par l'inflammasome; absence de peptide signal de sécrétion, l'empêchant d'utiliser la voie classique d'exocytose ; compétition extracellulaire avec un antagoniste naturel (IL-IRa); fixation extracellulaire sur le récepteur IL-1R2, qui ne contient pas de domaine cytoplasmique permettant la transduction du signal.

Du fait de ces différents mécanismes, il y a un découplage entre les niveaux de transcription de la pro-IL-1 $\beta$ et les niveaux de sécrétion de sa forme active. 


\begin{tabular}{|c|c|c|c|c|c|}
\hline Récepteurs & Stimulus & $\begin{array}{l}\text { DAMP/ } \\
\text { PAMP }\end{array}$ & $\begin{array}{c}\text { Interaction } \\
\text { directe/indirecte }\end{array}$ & $\begin{array}{l}\text { Transduction } \\
\text { du signal }\end{array}$ & Références \\
\hline \multirow[t]{2}{*}{ NLRPI } & Toxine létale de l'anthrax & PAMP & D & CARD-CARD & {$[35,36]$} \\
\hline & Muramyl dipeptide & PAMP & $\mathrm{D}$ & CARD-CARD & [34] \\
\hline \multirow[t]{10}{*}{ NLRP3 } & Toxines formant des pores & PAMP & 1 & & {$[15,23]$} \\
\hline & ATP extracellulaire via P2X7 & DAMP & 1 & & {$[15,16,22]$} \\
\hline & Dérivés réactifs de l'oxygène & DAMP & । & & {$[21,24]$} \\
\hline & Efflux d'ions $\mathrm{K}_{+}$ & DAMP & 1 & & [15-17] \\
\hline & Cristaux d'urate & DAMP & 1 & & [8] \\
\hline & Fibres d'amiante & DAMP & । & PYD-ASC-CARD & [19] \\
\hline & Cristaux de silice & DAMP & । & & {$[7,19]$} \\
\hline & Sels d'aluminium & DAMP & I & & [7] \\
\hline & Cristaux de cholestérol & DAMP & 1 & & [6] \\
\hline & Protéine $\beta$-amyloïde & DAMP & I & & [20] \\
\hline NLRP6 & $?$ & $?$ & $?$ & $?$ & {$[37,38]$} \\
\hline NLRP7 & Lipopeptide bactérien & PAMP & D & PYD-ASC-CARD & [39] \\
\hline NLRP12 & Motif inconnu de Yersinia pestis & PAMP & $?$ & PYD-ASC-CARD & {$[40]$} \\
\hline \multirow[t]{2}{*}{ NLRC4 } & Flagelline & PAMP & D & CARD-CARD & {$[27,33]$} \\
\hline & Systèmes de sécrétion de type 3 et 4 & PAMP & D & CARD-CARD & {$[27,33]$} \\
\hline AIM2 & ADN bactérien et viral & PAMP & D & PYD-ASC-CARD & {$[41,45,46]$} \\
\hline$|F| 16$ & ADN viral nucléaire & PAMP & D & PYD-ASC-CARD & [48] \\
\hline RIG-I* & ARN viral & PAMP & D & CARD-CARD ou ASC? & [13] \\
\hline
\end{tabular}

Tableau I. Principaux activateurs des inflammasomes. *Le rôle de RIG-I en tant qu'activateur de l'inflammasome reste débattu.

\section{Inflammasomes non canoniques}

L'inflammasome canonique est défini comme l'inflammasome conduisant à l'activation de la caspase-1. Récemment, deux voies de signalisation impliquant des caspases inflammatoires ou des acteurs de l'inflammasome, mais ne conduisant pas à l'activation de la caspase- 1 , ont été identifiées et nommées inflammasomes non canoniques. Le premier, décrit chez la souris, implique la caspase-11. Les mécanismes d'activation de cet inflammasome sont encore mal connus, mais impliqueraient la voie de l'interféron et des récepteurs encore inconnus pouvant détecter des bactéries intracytoplasmiques. L'activation de la caspase-11 conduit à la pyroptose et au relargage de nombreux DAMP (IL-1, HMGBI [high mobility group protein Bl], etc.). Le deuxième inflammasome non canonique implique l'adaptateur de l'inflammasome canonique, ASC, qui recrute et active la caspase-8, une caspase apoptotique. Cette voie est impliquée dans la réponse Fas/FasL, et dans la détection de champignons, de levures et de mycobactéries par des récepteurs extracellulaires. De plus, elle constitue une voie alternative à la caspase-l lors d'infections bactériennes.

\section{L'interleukine-18}

Contrairement à I'IL-1 $\beta$, I'IL-18 est exprimée constitutivement dans les macrophages et ne requiert donc pas d'étape de priming [31]. Une des principales fonctions de l'IL-18 est l'induction d'une réponse Thl, via l'activation des cellules natural killer (NK) et la sécrétion d'IFN- $\gamma$. L'IL-18 favorise aussi la sécrétion d'autres cytokines pro-inflammatoires, comme le TNF$\alpha$, I'IL-1 $\beta$, I'IL-8 et le GM-CSF (granulocyte-macrophage colony-stimulating factor). Ainsi, I'IL-18 induit le recrutement, l'activation et l'expansion des polynucléaires neutrophiles et des macrophages au cours des infections. En outre, l'IL-18 augmente l'activité cytotoxique et la prolifération des lymphocytes T CD8 ${ }^{+}$ et des cellules NK. Le rôle protecteur de l'IL-18 au cours des infections bactériennes et fongiques est principalement lié à sa capacité à entraîner la production d'IFN- $\gamma$, une cytokine qui induit l'activité microbicide 


\begin{tabular}{|c|c|c|c|}
\hline Inflammasomes & Bactéries & Motifs activateurs & Références \\
\hline NLRPl & Bacillus anthracis & Toxine létale & {$[35,36]$} \\
\hline \multirow[t]{13}{*}{ NLRP3 } & Burkholderia pseudomallei & Bsak & \\
\hline & Chlamydia pneumoniae & $?$ & \\
\hline & Escherichia coli & ARNm & [22] \\
\hline & Streptococcus B & Hémolysine $\beta$ & [23] \\
\hline & Listeria monocytogenes & Lystériolysine 0 & \\
\hline & Mycobacterium tuberculosis & ESX-1, हSAT-6 & \\
\hline & Staphylococcus aureus & Hémolysine $\alpha$ & [22] \\
\hline & Streptococcus pneumoniae & Pneumolysine & \\
\hline & Vibrio cholera & Toxine cholérique B & \\
\hline & yersinia pestis & Système de sécrétion de type 3 , YopJ & \\
\hline & Neisseria gonorrhoeae & $?$ & \\
\hline & Treponema denticola & $\mathrm{Td} 92$ & \\
\hline & Leptospira interrogans & Glycolipoprotéine & \\
\hline NLRP7 & Mycoplasma spp & Lipopeptide acylé & \\
\hline NLRP12 & Yersinia pestis & $?$ & {$[40]$} \\
\hline \multirow[t]{7}{*}{ NLRC4 } & Burkholderia pseudomallei & Bsak & \\
\hline & Escherichia coli & EprJ, Escl & \\
\hline & Legionella pneumophila & Flagelline & {$[27,33]$} \\
\hline & Pseudomonas aeruginosa & Flagelline, PscL & \\
\hline & Salmonella typhimurium & Système de sécrétion de type 3 , flagelline, PrgJ & \\
\hline & Shigella flexneri & Mxil & \\
\hline & Yersinia pestis & Système de sécrétion de type 3 & \\
\hline \multirow[t]{3}{*}{ AIM2 } & Francisella tularensis & ADN & {$[41]$} \\
\hline & Listeria monocytogenes & ADN & \\
\hline & Mycobacterium tuberculosis & ADN & \\
\hline
\end{tabular}

Tableau II. Bactéries activatrices des différents inflammasomes et motifs activateurs. ESX-1: ESAT-6 secretion system 1 ; ESAT-6:6-kDa early secreted antigenic target; YopJ : Yersinia outer protein J ; Mxil : Max-interactor 1.

des neutrophiles et des macrophages par de nombreux mécanismes antibactériens (exemple: production d'oxyde nitrique).

\section{La pyroptose}

Outre sa fonction activatrice d'interleukines pro-inflammatoires, la caspase- 1 est capable d'engager la cellule dans un processus de mort inflammatoire programmée, appelée pyroptose, restreinte à certains types cellulaires (dont les macrophages, les cellules dendritiques, ou les neurones) [4, 32]. La pyroptose est déclenchée en réponse à de nombreux stimulus, qu'il s'agisse de PAMP ou de DAMP. II s'agit d'une mort cellulaire rapide, dépendante de la caspase-1, et caractérisée par une rupture de la membrane plasmique et la libération consécutive des substances pro-inflammatoires intracellulaires. En effet, la caspase-1 activée induit la formation de pores dans la membrane cellulaire, condui- 


\begin{tabular}{|c|c|c|c|}
\hline Inflammasomes & Virus & $\begin{array}{l}\text { ARN/ } \\
\text { ADN }\end{array}$ & $\begin{array}{l}\text { Motifs } \\
\text { activateurs }\end{array}$ \\
\hline \multirow[t]{10}{*}{ NLRP3 } & Virus de la rougeole & ARN & $?$ \\
\hline & Virus de l'hépatite C & ARN & $?$ \\
\hline & Virus influenza & ARN & Insertion de M2 \\
\hline & $\begin{array}{l}\text { Virus respiratoire } \\
\text { syncitial }\end{array}$ & ARN & ROS/efflux de K+ \\
\hline & Virus de la dengue & ARN & CLEC5A \\
\hline & MVA & ADN & $?$ \\
\hline & Myxoma virus & ADN & $\begin{array}{l}\text { Cathepsine B/ } \\
\text { ROS }\end{array}$ \\
\hline & Virus varicelle-zona & ADN & $?$ \\
\hline & Herpes simplex 1 & ADN & $?$ \\
\hline & VIH l & ADN & $?$ \\
\hline \multirow[t]{3}{*}{ IFII6* } & Herpes simplex 1 & ADN & ADNdb? \\
\hline & KSHV & ADN & ADNdb? \\
\hline & Cytomégalovirus & ADN & ADNdb? \\
\hline RIG-I* & $?$ & ARN? & ARNsb? \\
\hline
\end{tabular}

Tableau III. Virus activateurs des inflammasomes. *Les rôles directs de RIG-I et IFI16 en tant qu'activateurs de l'inflammasome restent à confirmer. CLEC5A : C-type lectin $5 A ; \mathrm{VIH}$ : virus de l'immunodéficience humaine; $\mathrm{db}$ : double brin ; sb : simple brin.

sant ainsi à une augmentation de la pression osmotique, un afflux d'eau à l'intérieur de la cellule et un gonflement cellulaire. Ces étapes aboutissent à la lyse osmotique de la cellule et au relargage des composants inflammatoires vers le compartiment extracellulaire. Contrairement à l'apoptose, ce processus est marqué par une fragmentation de l'ADN non oligonucléosomal, une condensation nucléaire sans perte d'intégrité du noyau et une destruction du cytosquelette d'actine.

L'importance de ce processus est particulièrement flagrante au cours des infections à bactéries intracellulaires, dont Legionella pneumophila. En effet, après l'invasion de la cellule par une légionelle, celle-ci va s'établir au sein d'une vacuole de réplication intracytoplasmique où, protégée des défenses immunitaires de l'hôte, elle va effectuer de nombreux cycles de multiplication. Dans les macrophages, la détection de la flagelline de Legionella par NLRC4 induit la formation de l'inflammasome. L'activation de la caspase-l et la pyroptose qui en résulte permettent, non seulement la destruction précoce du terrain de réplication de la bactérie (mort de la cellule), mais aussi l'induction d'un phénomène inflammatoire local (recrutement d'autres cellules immunitaires) concourant au confinement de l'infection [33].

\section{L'inflammasome au cours des maladies infectieuses}

L'importance de l'activation de l'inflammasome dans la lutte contre les infections résulte des trois activités de la caspase-1: (1) la pyroptose, qui permet la suppression des niches de réplication des bactéries intracellulaires; (2) la production d'IL-1 $\beta$, qui entraîne le recrutement des cellules inflammatoires au site de l'infection; et (3) la production d'IL-18, qui induit la cascade de l'IFN- $\gamma$ et l'activation des mécanismes bactéricides cellulaires. Nous présentons ci-contre les mécanismes d'activation de l'inflammasome au cours de différentes infections bactériennes (Tableau II) et virales (Tableau III).

\section{Infections bactériennes}

Chez l'homme, l'inflammasome NLRPl est activé en présence de muramyl dipeptide, un fragment du peptidoglycane présent dans la paroi de nombreuses bactéries à Gram positif comme à Gram négatif [34]. Par ailleurs, chez la souris, le facteur létal, un des deux constituants de la toxine létale du bacille de l'anthrax (Bacillus anthracis), est également reconnu par NLRP1 [35]. L'inflammasome a un rôle central dans la lutte contre $B$. anthracis dans des modèles murins, mais pourrait aussi être responsable des dommages pulmonaires inflammatoires en cas d'inhalation de la toxine létale [36]. Cette action paradoxale permet de bien comprendre la nécessité de contrôler l'inflammasome, et l'inflammation en règle générale, pour lutter contre les microbes sans léser les tissus de l'hôte.

NLRP3 est activé par de nombreuses bactéries en réponse à des signaux de danger plutôt que par une interaction directe PAMP-récepteur. Les différentes voies d'activation de NLRP3 pouvant être engagées ont été détaillées ci-dessus. Les bactéries activant NLRP3, ainsi que leur motif activateur, sont détaillées dans le Tableau II. À titre d'exemple, au cours des infections cutanées à $S$. aureus, l'activation de l'inflammasome NLRP3 résultant de la détection des dommages membranaires causés par les toxines formant des pores, est déterminante pour entraîner le recrutement précoce des neutrophiles au site d'infection, via la sécrétion d'IL-1B.

Le rôle de NLRP6 dans l'immunité antibactérienne a été rapporté récemment, mais de manière contradictoire et uniquement dans des modèles murins. En effet, dans un cas, les souris déficientes en NLRP6 présentaient une altération de la flore microbienne intestinale et une prédisposition pour les colites [37]. Dans l'autre cas, ces souris semblaient résistantes aux infections par Listeria monocytogenes, Salmonella et Escherichia coli [38]. Au total, le (ou les) activateur(s) de NLRP6 demeure(nt) inconnus, et son (leur) rôle reste à caractériser.

Le rôle antibactérien de NLRP7 et NLRP12 a été décrit. NLRP7 semble impliqué dans la reconnaissance de lipopeptides bactériens provenant d'espèces de Myco- 
plasma [39], mais il est mal caractérisé du fait de son absence dans le modèle murin. NLRP12, quant à lui, forme un inflammasome en réponse à l'infection par Yersinia, mais cette observation n'a été faite que dans un modèle murin, et le motif détecté n'est pas identifié [40].

NLRC4 est spécifiquement activé par les systèmes de sécrétion de type III et IV de bactéries, comme Yersinia et Shigella, ou par la flagelline des bactéries telles que Salmonella, Legionella, ou Pseudomonas $[27,33]$. Dans les modèles murins, la spécificité de l'inflammasome NLRC4 pour un de ses activateurs semble être conférée par la liaison préalable de l'activateur à l'une ou l'autre des protéines NAIP [29]. Cependant, seule une protéine NLRB (homologue de NAIP) est présente chez l'homme et, même si elle semble capable de reconnaître un motif minimal commun à Shigella, Salmonella et Pseudomonas, des études complémentaires sont nécessaires pour confirmer le rôle de NLRB. Chez la souris, le complexe NLRB/NLRC4 a un rôle clé dans la détection précoce de l'invasion bactérienne au niveau de la muqueuse intestinale et dans le déclenchement d'une inflammation forte permettant le contrôle de l'infection.

AIM2 reconnaît spécifiquement l'ADN double brin présent dans le cytoplasme. Il est activé au cours des infections bactériennes ou virales lorsque l'agent pathogène se trouve en dehors du phagosome, en particulier dans les infections à Listeria monocytogenes, l'agent de la listériose, ou à Francisella tularensis, l'agent de la tularémie. Ainsi, les souris déficientes pour AIM2 sont très susceptibles à l'infection par Francisella [41].

De façon intéressante, le récepteur AIM2 détecte une molécule commune aux virus et à tous les êtres vivants, y compris l'homme. Le signal de danger n'est donc pas la détection de cette molécule commune, I'ADN, mais plutôt la détection de sa mauvaise localisation. En effet, AIM2 détecte I'ADN présent dans le cytosol cellulaire alors que, dans une cellule saine, la localisation de l'ADN est restreinte au noyau.

\section{Infections virales}

Le rôle des cytokines pro-inflammatoires IL-1 $\beta$ et IL-18 dans l'immunité antivirale a été démontré dans les modèles murins. Par exemple, au cours de l'infection par le virus de la grippe, la mortalité est supérieure chez les souris déficientes en IL-1R ou en IL-18 par rapport aux souris sauvages $[42,43]$. Par ailleurs, l'infection par le virus herpès de type 1 (HSVI) de souris déficientes en IL-l résulte en une charge virale sanguine augmentée, un taux d'encéphalites à HSVl plus élevé, et ce dans un contexte moins inflammatoire. L'administration d'IL-18 à un stade précoce de l'infection par HSVl entraîne une augmentation de la survie des souris.

Un modèle intéressant d'activation de l'inflammasome NLRP3 au cours de l'infection par le virus de la grippe A (influenza A) a été proposé récemment. Au niveau des macrophages pulmonaires, la reconnaissance de l'ARN viral par le récepteur TLR7 produirait le premier signal conduisant à la synthèse de pro-IL-1 $\beta$. Le second signal serait ensuite représenté par l'insertion de la protéine virale M2 (un canal ionique) au niveau du réseau transgolgien, entraînant des perturbations du milieu ionique intracellulaire. Une autre possibilité serait la survenue de dommages lysosomaux liés à la pénétration du virus et associés à un efflux de potassium et à une production de ROS, déclenchant l'activation de NLRP3 [44].

Les données concernant l'activation de l'inflammasome AIM2 au cours des infections virales sont rares et se concentrent essentiellement sur les modèles murins. Le cytomégalovirus murin, un virus à ADN de la famille des herpesviridae, est reconnu par AIM2 [45, 46]. Au contraire, d'autres herpesviridae, HSVI et VZV (virus varicelle-zona), n'activent pas l'inflammasome AIM2, mais plutôt NLRP3 (HSVl et VZV) et IFII6 (HSVI) [47, 48].

La localisation intranucléaire d'IFll6 explique son activation par I'ADN des virus KSHV (Kaposi's sarcomaassociated herpes virus) et HSVI, dont la réplication s'effectue à l'intérieur du noyau.

À l'heure actuelle et à notre connaissance, aucune donnée ne permet cependant de statuer sur la survenue d'une mort cellulaire pyroptotique en réponse à une infection virale.

\section{Conclusion}

Dix ans après la découverte de l'inflammasome et malgré les grandes avancées dans la compréhension de ce complexe de l'immunité innée, ses mécanismes d'activation, de régulation et ses fonctions, notamment en lien avec les cellules de l'immunité adaptative, sont loin d'être totalement définis. Par ailleurs, il reste très vraisemblablement de nouveaux inflammasomes à découvrir. Bien que les mécanismes moléculaires des inflammasomes soient de mieux en mieux connus, les rôles physiopathologiques de l'inflammasome sont encore très mystérieux. S'il est bien démontré que I'inflammasome exerce un effet protecteur au cours des infections par de nombreuses bactéries, son rôle dans les infections virales, fongiques et parasitaires reste à confirmer.

Par ailleurs, l'inflammasome est un inducteur très puissant de réponses inflammatoires dont les effets peuvent être dévastateurs. Ces effets délétères de l'inflammation impliquant l'inflammasome sont particulièrement frappants chez les patients atteints de cryopyrinopathies, des maladies auto-inflammatoires héréditaires causées par des mutations sur le gène NLRP3. Ces maladies pédiatriques sont l'urticaire familial au froid, le syndrome de Muckle-Wells et le syndrome NOMID. Chez les patients porteurs de telles mutations, la dérégulation de l'inflammasome, du fait de la synthèse d'un récepteur NLRP3 spontanément activé, provoque une hyperproduction d'IL-1 $\beta$ et un état hyperinflammatoire récurrent se traduisant par des symptômes variant de la simple éruption cutanée 
jusqu'à la mise en jeu du pronostic vital. Le traitement par des antagonistes de l'IL-1, et particulièrement l'administration d'un antagoniste du récepteur de l'IL-1 (IL-1Ra, anakinra), a révolutionné la prise en charge de ces pathologies. D’autres maladies sémiologiquement proches des cryopyrinopathies, comme la maladie de Still de l'enfant ou de l'adulte ou encore le syndrome de Schnitzler, pourraient aussi impliquer des dérégulations de l'inflammasome et donc bénéficier de traitements ciblant cette voie.

Les recherches présentes et futures devraient permettre le décryptage complet des voies de l'inflammasome et de ses fonctions physiopathologiques et entraîner, de facto, la mise au point de thérapies ciblées utilisables dans nombre de situations infectieuses ou auto-inflammatoires, et éventuellement dans le cancer $(\rightarrow)[53]$. $\diamond$

\section{$(\rightarrow)$ Voir la Synthèse de J. Garaude, page 985 de ce numéro}

\section{SUMMARY}

\section{The inflammasomes: platforms of innate immunity}

Human beings are constantly exposed to pathogens. The innate immune system is the first line of defense against microbes. It has evolved to recognize conserved microbial motifs (PAMP or pathogen-associated molecular patterns) thanks to a limited array of receptors termed pattern recognition receptors (PRR). Upon activation, most PRR trigger a transcriptional response leading to neosynthesis of hundreds of genes. In contrast, engagement of various PRR in the recently identified inflammasome complexes lead to activation of a cysteine protease, caspase-1. This inflammatory caspase has a dual activity: it triggers the release of very potent proinflammatory cytokines IL- $1 \beta$ and IL-18 and, an hyperinflammatory cell death termed pyroptosis. In this review, we describe the inflammasome receptors and their ligands, the molecular mechanisms leading to the assembly of this innate immune platform and the role of the inflammasome during viral and bacterial infections. $\diamond$

\section{LIENS D'INTÉRÊT}

Les auteurs déclarent n'avoir aucun lien d'intérêt concernant les données publiées dans cet article.

\section{RéFÉRENCES}

1. Medzhitov R, Janeway CA Jr. Decoding the patterns of self and nonself by the innate immune system. Science $2002 ; 296: 298-300$.

2. Matzinger P. The danger model : a renewed sense of self. Science $2002 ; 296: 301-5$.

3. Martinon F, Burns K, Tschopp J. The inflammasome : a molecular platform triggering activation of inflammatory caspases and processing of prolL-beta. Mol Cell $2002 ; 10: 417-26$.

4. Bergsbaken T, Fink SL, Cookson BT. Pyroptosis : host cell death and inflammation. Nat Rev Microbiol $2009 ; 7: 99-109$.

5. Ting JPY, Lovering RC, Alnemri ES, et al. The NLR gene family : a standard nomenclature. Immunity $2008 ; 28: 285-7$.

6. Duewell P, Kono H, Rayner KJ, et al. NLRP3 inflammasomes are required for atherogenesis and activated by cholesterol crystals. Nature $2010 ; 464$ : 1357-61.

7. Hornung V, Bauernfeind F, Halle A, et al. Silica crystals and aluminum salts activate the NALP3 inflammasome through phagosomal destabilization. Nat Immunol 2008 ; 9 : 847-56.

8. Martinon F, Pétrilli V, Mayor A, et al. Gout-associated uric acid crystals activate the NALP3 inflammasome. Nature $2006 ; 440: 237-41$.

9. Schattgen SA, Fitzgerald KA. The PYHIN protein family as mediators of host defenses. Immunol Rev $2011 ; 243: 109-18$.

10. Fernandes-Alnemri T, $\mathrm{Yu}$ J-W, Datta $\mathrm{P}$, et al. AIM2 activates the inflammasome and cell death in response to cytoplasmic DNA. Nature $2009 ; 458$ : 509-13.
11. Kerur N, Veettil MV, Sharma-Walia N, et al. IFIl6 acts as a nuclear pathogen sensor to induce the inflammasome in response to Kaposi sarcomaassociated herpesvirus infection. Cell Host Microbe 2011 ; 9 : 363-75.

12. Satoh T, Kato H, Kumagai Y, et al. LGP2 is a positive regulator of RIG-I- and MDA5-mediated antiviral responses. Proc Natl Acad Sci USA 2010 ; 107 : 1512-7.

13. Poeck H, Bscheider M, Gross 0 , et al. Recognition of RNA virus by RIG-I results in activation of CARD9 and inflammasome signaling for interleukin 1 beta production. Nat Immunol $2010 ; 11: 63-9$.

14. Py BF, Kim MS, Vakifahmetoglu-Norberg H, et al. Deubiquitination of NLRP3 by BRCC3 critically regulates inflammasome activity. Mol Cell 2013; 49 : 331-8.

15. Mariathasan S, Weiss DS, Newton K, et al. Cryopyrin activates the inflammasome in response to toxins and ATP. Nature $2006 ; 440: 228-32$.

16. Kanneganti TD, Lamkanfi M, Kim YG, et al. Pannexin-1-mediated recognition of bacterial molecules activates the cryopyrin inflammasome independent of Toll-like receptor signaling. Immunity 2007 ; 26 : 433-43.

17. Pétrilli V, Papin S, Dostert C, et al. Activation of the NALP3 inflammasome is triggered by low intracellular potassium concentration. Cell Death Differ $2007 ; 14: 1583-9$.

18. Compan V, Baroja-Mazo A, López-Castejón G, et al. Cell volume regulation modulates NLRP3 inflammasome activation. Immunity $2012 ; 37$ : 487-500.

19. Dostert C, Pétrilli V, Bruggen R Van, et al. Innate immune activation through Nalp3 inflammasome sensing of asbestos and silica. Science 2008; 320 : 674-7.

20. Halle A, Hornung V, Petzold GC, et al. The NALP3 inflammasome is involved in the innate immune response to amyloid-beta. Nat Immunol $2008 ; 9: 857-65$.

21. Bauernfeind F, Ablasser A, Bartok $\varepsilon$, et al. Inflammasomes : current understanding and open questions. Cell Mol Life Sci $2011 ; 68$ : 765-83.

22. Franchi L, Kanneganti T-D, Dubyak GR, et al. Differential requirement of $\mathrm{P} 2 \mathrm{X7}$ receptor and intracellular $\mathrm{K}^{+}$for caspase- 1 activation induced by intracellular and extracellular bacteria. J Biol Chem $2007 ; 282$ : 18810-8.

23. Costa A, Gupta R, Signorino G, et al. Activation of the NLRP3 inflammasome by group B streptococci. J Immunol $2012 ; 188$ : 1953-60.

24. Tschopp J, Schroder K. NLRP3 inflammasome activation: The convergence of multiple signalling pathways on ROS production? Nat Rev Immunol 2010; $10: 210-5$.

25. Zhou R, Tardivel A, Thorens B, et al. Thioredoxin-interacting protein links oxidative stress to inflammasome activation. Nat Immunol 2010 ; $11: 136-40$.

26. Shimada K, Crother TR, Karlin J, et al. Oxidized mitochondrial DNA activates the NLRP3 inflammasome during apoptosis. Immunity 2012 ; $36: 401-14$.

27. Coers J, Vance RE, Fontana MF, et al. Restriction of Legionella pneumophila growth in macrophages requires the concerted action of cytokine and Naip5/Ipaf signalling pathways. Cell Microbiol 2007 ; 9 : 2344-57.

28. Abdelaziz DHA, Gavrilin MA, Akhter A, et al. Asc-dependent and independent mechanisms contribute to restriction of legionella pneumophila infection in murine macrophages. Front Microbiol $2011 ; 2$ : 18.

29. Kofoed EM, Vance RE. NAIPs : building an innate immune barrier against bacterial pathogens. NAIPs function as sensors that initiate innate immunity by detection of bacterial proteins in the host cell cytosol. Bioessays $2012 ; 34$ : 589-98.

30. Dinarello CA. Interleukin-1 in the pathogenesis and treatment of inflammatory diseases. Blood $2011 ; 117: 3720-32$.

31. Sahoo M, Ceballos-Olvera I, Barrio L del, et al. Role of the inflammasome, IL-1 $\beta$, and IL-18 in bacterial infections. Sci World J $2011 ; 11$ : 2037-50.

32. Miao EA, Rajan JV, Aderem A. Caspase-1-induced pyroptotic cell death. Immunol Rev $2011 ; 243$ : 206-14.

33. Jamilloux $Y$, Jarraud $S$, Lina G, et al. Legionella, légionnellose. Med Sci (Paris) $2012 ; 28: 639-45$.

34. Bruey J-M, Bruey-Sedano N, Luciano F, et al. Bcl-2 and Bcl-XL regulate proinflammatory caspase-1 activation by interaction with NALP1. Cell 2007 ; $129: 45-56$.

35. Terra JK, Cote CK, France B, et al. Cutting edge : resistance to Bacillus anthracis infection mediated by a lethal toxin sensitive allele of Nalplb/ Nlrplb. J Immunol 2010 ; 184 : 17-20.

36. Kovarova M, Hesker PR, Jania L, et al. NLRPl-dependent pyroptosis leads to acute lung injury and morbidity in mice. J Immunol 2012 ; 189 : 2006-16.

37. Elinav $\varepsilon$, Strowig T, Kau AL, et al. NLRP6 inflammasome regulates colonic microbial ecology and risk for colitis. Cell $2011 ; 145$ : 745-57.

38. Anand PK, Malireddi RKS, Lukens JR, et al. NLRP6 negatively regulates innate immunity and host defence against bacterial pathogens. Nature $2012 ; 488$ : 389-93. 


\section{RÉFÉRENCES}

39. Khare S, Dorfleutner A, Bryan NB, et al. An NLRP7-containing inflammasome mediates recognition of microbial lipopeptides in human macrophages. Immunity $2012 ; 36: 464-76$.

40. Vladimer GI, Weng D, Paquette SWM, et al. The NLRP12 inflammasome recognizes Yersinia pestis. Immunity $2012 ; 37$ : 96-107.

41. Jones JW, Kayagaki N, Broz P, et al. Absent in melanoma 2 is required for innate immune recognition of Francisella tularensis. Proc Natl Acad Sci USA 2010 ; 107 : 9771-6.

42. Schmitz N, Kurrer M, Bachmann MF, et al. Interleukin-l is responsible for acute lung immunopathology but increases survival of respiratory influenza virus infection. J Virol $2005 ; 79$ 6441-8.

43. Liu B, Mori I, Hossain MJ, et al. Interleukin-18 improves the early defence system against influenza virus infection by augmenting natural killer cell-mediated cytotoxicity. J Gen Virol 2004 ; 85 : 423-8.

44. Ichinohe T, Pang IK, Iwasaki A. Influenza virus activates inflammasomes via its intracellular M2 ion channel. Nat Immunol $2010 ; 11: 404-10$.

45. Hornung V, Ablasser A, Charrel-Dennis M, et al. AIM2 recognizes cytosolic dsDNA and forms a caspase-1-activating inflammasome with ASC. Nature $2009 ; 458: 514-8$.

46. Rathinam VAK, Fitzgerald KA. Inflammasomes and anti-viral immunity. J Clin Immunol 2010 ; 30 : 632-7.

47. Nour AM, Reichelt $M, K u C-C$, et al. Varicella-zoster virus infection triggers formation of an interleukin-1 $\beta$ (IL-1 $\beta$ )-processing inflammasome complex. J Biol Chem 2011 ; 286 : 17921-33.
48. Muruve DA, Pétrilli V, Zaiss AK, et al. The inflammasome recognizes cytosolic microbial and host DNA and triggers an innate immune response. Nature $2008 ; 452: 103-7$.

49. Imler JL, Ferrandon D. Le printemps de l'immunité innée couronné à Stockholm. Med Sci (Paris) $2011 ; 27$ : 1019-1024.

50. Bisbal C, Salehzada T. La RNase L, un acteur essentiel de la réponse cellulaire antivirale. Med Sci (Paris) $2008 ; 24: 859-64$.

51. Kowalinski $\varepsilon$, Louber J, Gerlier D, Cusack S. RIG-I. Un commutateur moléculaire détecteur d'ARN viral. Med Sci (Paris) $2012 ; 28: 136-8$.

52. Pochet $S$, Seil M, El Ouaaliti M, Dehaye JP. $P 2 X_{4}$ ou $P 2 X_{7}$ : lequel de ces deux récepteurs nous fera saliver? Med Sci (Paris) $2013 ; 29$ : 509-14.

53. Garaude J. Levée de l'immunité innée dans le traitement des cancers. Med Sci (Paris) $2013 ; 29: 985-90$.

54. Pellé KG, Ahouidi AD, Mantel Py. Le rôle des microvésicules dans l'infection palustre. Med Sci (Paris) 2013 ; 29 : 960-2.

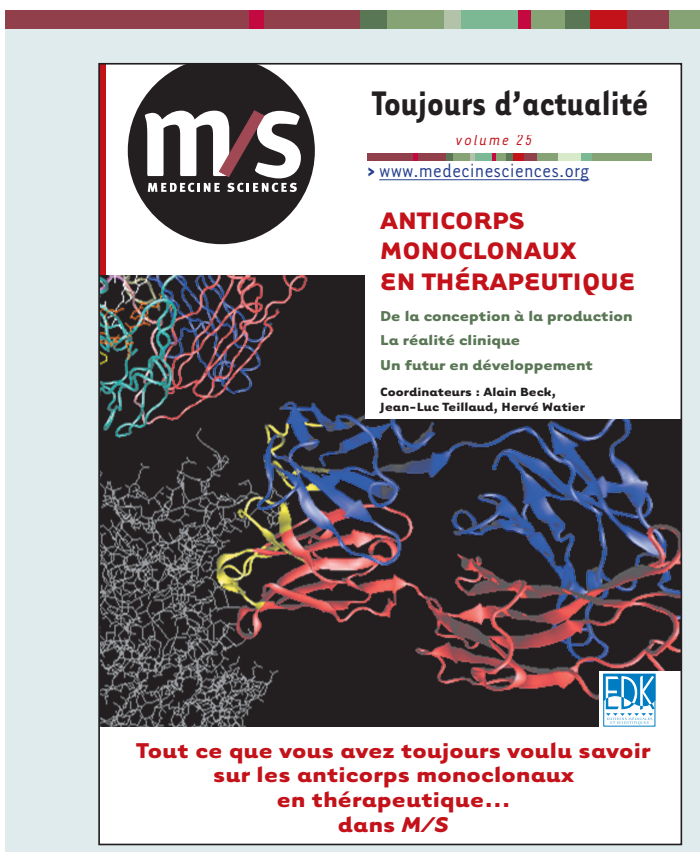

Tout ce que vous avez toujours voulu savoir sur les anticorps monoclonaux en thérapeutique... dans Médecine/Sciences. Pourquoi un numéro spécial de Médecine/Sciences sur les anticorps monoclonaux thérapeutiques? II nous a semblé que le moment était venu de dresser un état des lieux de ces biomédicaments qui prennent désormais une place considérable - et croissante dans les traitements de maladies souvent lourdes et désespérantes. Ce voyage que nous vous proposons à la découverte du monde des anticorps thérapeutiques nous a appris, ou plutôt rappelé, une évidence : les compétences en France sont fortes et nombreuses, qu'elles soient académiques ou industrielles, biotechnologiques ou cliniques. Le paysage français, trop longtemps discret, bruisse désormais de mille initiatives balayant de multiples aspects des anticorps thérapeutiques : études précliniques et cliniques menées avec de nouveaux anticorps dirigés contre des cibles originales, développement de nouveaux formats d'anticorps ou d'anticorps optimisés reposant sur des études structurales et fonctionnelles sophistiquées, recherche active de cibles pertinentes, mise au point de méthodologies de bioproduction, de couplage, etc. L'expansion industrielle rapide de ce champ est un défi que peut et doit relever notre pays, défi tant scientifique qu'économique, avec ses combats pour la propriété intellectuelle et pour l'emploi de nos jeunes scientifiques.

Alain Beck, Jean-Luc Teillaud, Hervé Watier

À retourner à EDK, 25, rue Daviel - 75013 Paris, France

Tél. : 0158101905 - Fax : 0143293262 - E-mail : edk@edk.fr

NOM :

Prénom :

Adresse :

Code postal :

Ville :

Pays :

Fonction :

Je souhaite recevoir $\mathbf{M} / \mathbf{S} \mathbf{n}^{\circ} \mathbf{1 2}$ - décembre 2009 (Anticorps monoclonaux en thérapeutique) : $25 €+3 €$ de port $=\mathbf{2 8} €$ TTC en exemplaire, soit un total de $€$

Par chèque, à l'ordre de $\mathbf{E} \mathbf{D} \mathbf{K}$

Par carte bancaire : $\square$ Visa

Eurocard/Mastercard

Carte $n^{\circ}$

Date d'expiration :

$\mathrm{N}^{\circ}$ de contrôle au dos de la carte :

Signature : 